\title{
Diagnostic value of KLK6 as an ovarian cancer biomarker: A meta-analysis
}

\author{
FAN YANG ${ }^{1}$, ZHI-DE HU ${ }^{2}$, YINGJIAN CHEN $^{2}$ and CHENG-JIN HU ${ }^{2}$ \\ ${ }^{1}$ Graduate School of Jinzhou Medical University, Jinzhou, Liaoning 121001; ${ }^{2}$ Department of Laboratory Medicine, \\ General Hospital of Ji'nan Military Command Region, Ji'nan, Shandong 250031, P.R. China
}

Received January 15, 2016; Accepted April 5, 2016

DOI: $10.3892 /$ br.2016.662

\begin{abstract}
Kallikrein-related peptidase 6 (KLK6) is a new potential serum biomarker of ovarian cancer. The aim of the present study was to assess the diagnostic value of KLK6 systematically for ovarian cancer. All the selected studies regarding the changes of KLK6 in ovarian cancer were published prior to April 2015. Five studies involving 485 patients with ovarian cancer, 420 benign cysts and 245 healthy controls met the inclusion criteria. The value of sensitivity, specificity, positive-likelihood ratio (LR+), negative-likelihood ratio (LR-) and area under the receiver operating characteristic curve (ROC) were obtained. All these indices were used to evaluate the diagnostic value of KLK6 for ovarian cancer. The values of sensitivity, specificity, LR+ and LR- (95\% confidence interval) of KLK6 were 0.50 (0.47-0.54), 0.91 (0.89-0.93), 7.20 (3.34-15.52) and 0.51 (0.43-0.62), respectively. The area under the summary ROC of KLK6 was 0.86 . The index of $Q^{*}$ was 0.79 . In conclusion, KLK6 showed high specificity for the diagnosis of ovarian cancer. It can improve the diagnostic accuracy of cancer antigen 125 (CA125). A combined panel of CA125 and KL K6 shows a high diagnostic efficiency for advanced ovarian cancer. Owing to the small number of studies and lack of samples, additional studies meeting the inclusion criteria are required to further analyze the diagnostic value of KLK6 for ovarian cancer.
\end{abstract}

\section{Introduction}

Ovarian cancer is one of the major causes of cancer-associated fatalities among gynecological malignancies (1). In recent years, discovery and diagnosis of ovarian cancer at an early stage is limited, and there is no effective therapy at advanced stages. The gold standard for diagnosis of ovarian cancer is pathological evidence. Due to the invasive nature of this procedure, it is not suitable for screening patients of early-stage

Correspondence to: Dr Cheng-Jin Hu, Department of Laboratory Medicine, General Hospital of Ji'nan Military Command Region, 25 Shifan Road, Ji'nan, Shandong 250031, P.R. China

E-mail: hcj6289@163.com

Key words: kallikrein-related peptidase 6, ovarian cancer, diagnoses, meta-analysis cancer. Therefore, the identification of effective biomarkers for ovarian cancer is necessary.

Currently, cancer antigen 125 (CA125) is a well-accepted serum biomarker for ovarian cancer, but is neither specific nor sensitive enough for accurate diagnosis (2-4). Bast et al (5) detected CA125 in 1983. The serum CA125 level is also affected by numerous primary tumors and female benign diseases (BD). Therefore, it is necessary to identify novel biomarkers to improve the diagnostic efficiency for ovarian cancer and to monitor high-risk patients, such as those with breast cancer 1 (BRCA1) and BRCA2, infertility or menstrual disorders.

Kallikrein-related peptidase (KLK) (6) is a family of 15 members encoded by a group of genes tandemly localized on chromosome 19q13.3-4 (7). KLK6 is the protein that is encoded by the $K L K$ gene and one of the KLKs. The mature KLK6 consists of 223 amino acids with trypsin-like activity (8). It is highly expressed in reproductive organs such as the breast, ovary, prostate and testis. In 2000, KLK6 was verified in various biologic fluids, including cerebrospinal fluid, nipple aspirate fluid, breast cyst fluid, male and female serum, seminal plasma, amniotic fluid and breast cancer cytosols (9). A previous study showed that KLK6 is a new potential serum biomarker for diagnosis and prognosis of ovarian cancer (10). Serum KLK6 levels were shown to increase in patients with late-stage ovarian cancer with and without correlation to serum CA125 levels, respectively (11). There are numerous published studies that have evaluated the diagnostic value of KLK6; however, there are no consistent results. Therefore, a meta-analysis to ascertain the diagnostic value of KLK6 systemically is required.

The aim of the present study was to systematically assess whether the level of serum KLK6 could be used in the detection of ovarian cancer, with a view to aid in the diagnosis of ovarian cancer and choose the effective therapy.

\section{Materials and methods}

Screening process. Studies that were published before April 29, 2015, were screened on a number of platforms, such as PubMed/Medline, Embase, EBSCO, ScienceDirect, Cochrane library and Web of Science. All the studies were selected using keywords as follows: 'KLK6' (kallikrein-related peptidase 6), 'hk6' (human kallikrein 6), 'protease M,' 'zyme,' 'neurosin,' 'ovarian cancer' (OC), 'ovarian carcinoma,' 'oophoroma,' 'ovarian neoplasm' and 'ovarian neoplasms'. 
Study selection. The inclusion criteria were as follows: i) All the patients with ovarian cancer were diagnosed by pathology clearly; ii) the predictive value of serum KLK6 was obtained by enzyme-linked immunosorbent assay or chemiluminescent microparticle immunoassay; and iii) studies must have the following terms: False-negative (FN), false-positive (FP), true-negative (TN) and true-positive (TP).

The exclusion criteria were as follows: i) The information of the control group was not described clearly; ii) review, animal experiment and seminars were not included; and iii) the studies were not published in English or Chinese.

Data extraction and quality assessment. All five studies were reading intensively to obtain the number of ovarian cancer patients, non-ovarian cancer patients, the values of sensitivity (SEN) and specificity (SPE) reported. The values of FN, FP, TN, TP, positive-likelihood ratio (LR+), negative-likelihood ratio (LR-) were calculated according to the following formulas:

$\mathrm{TP}=$ Number of ovarian cancer patients $\mathrm{x}$ SEN

FN $=$ Number of ovarian cancer patients $x$ (1-SEN)

$\mathrm{TN}=$ Number of non-ovarian cancer patients $\mathrm{x}$ SPE

$\mathrm{FP}=$ Number of non-ovarian cancer patients $\mathrm{x}(1-\mathrm{SPE})$

$\mathrm{LR}+=\mathrm{SEN} /(1-\mathrm{SPE})$

LR- $=(1-\mathrm{SEN}) / \mathrm{SPE}(12)$

The blinded method was used by two investigators (ChengJin $\mathrm{Hu}$ and Fan Yang) for reviewing and screening the included studies. The widely used Quality Assessment of Diagnostic Accuracy Studies (QUADAS2) tool was applied to assess the quality of all included studies (13).

Statistical analysis. Meta-DiSc statistical software (version 1.4) was used to obtain all the data (14). Heterogeneity was checked by Spearman correlation analysis and meta-regression. The index of the P-value and inconsistency index (I-squared, $\mathrm{I}^{2}$ ) were used to estimate the heterogeneity. When there was no significant heterogeneity between studies $\left(\mathrm{P}>0.05, \mathrm{I}^{2} \leq 50 \%\right)$, the analysis was performed using a fixed-effects model and analyzed bias to obtain SEN, SPE, positive-predictive and negative-predictive values. When there was statistical heterogeneity among studies, the analysis was performed using the random-effects model $\left(\mathrm{P} \leq 0.05, \mathrm{I}^{2}>50 \%\right)$. The pooled SEN, pooled SPE, LR+, LR- and their $95 \%$ confidence interval (CI) were calculated using the DerSimonian-Laird random-effects method. The summary receiver operating characteristic (sROC), the area under the sROC curve (AUC) and the Q* value were calculated to estimate the diagnostic accuracy of index test in the meta-analysis. An AUC close to 1.0 signifies that the test has almost perfect discrimination, while an AUC close to 0.5 suggests poor discrimination. The index $\mathrm{Q}^{*}$ corresponds to the upper most point on the SROC curve in which true positivity (or SEN) equals SPE. The sROC summarized the joint distribution of SEN and SPE. This can be shown graphically by drawing a 'line of identity' in which true positivity equals SPE on the sROC graph (13). $\mathrm{P}<0.05$ by $\chi^{2}$ was considered to indicate a statistically significant difference.

\section{Results}

Included studies. Following intensive assessment of the 92 retrieved studies and taking the inclusion and exclusion

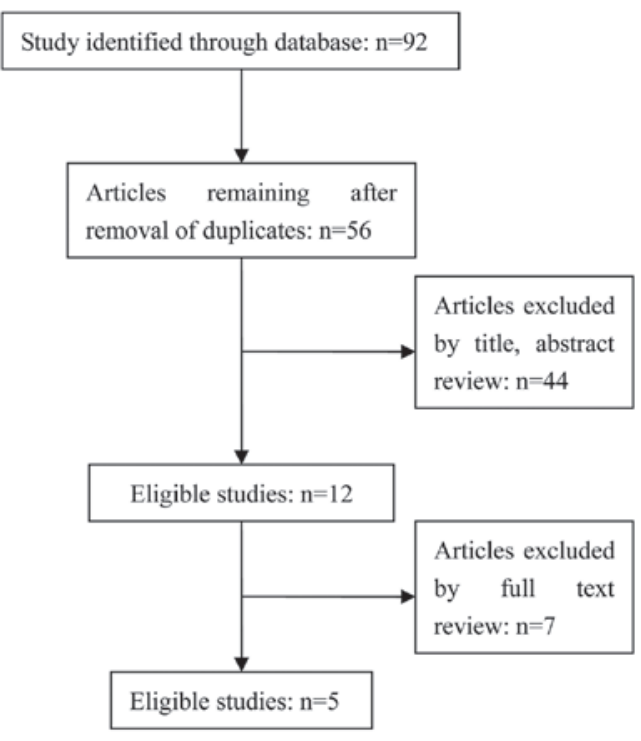

Figure 1. Flowchart of the systematic literature search and study selection process.

criteria into account, there were 5 studies selected $(8,10,15-17)$. A flowchart depicting the study selection is shown in Fig. 1. These 5 studies were published before 2015. The methodology quality of these 5 studies was assessed using QUADAS2 (the description of QUADAS2 is shown in Table I). In the 5 included studies there were 1,150 samples, and there were 485,420 and 245 patients with ovarian cancer, BD and the healthy controls, respectively. All the patients with ovarian cancer were diagnosed clearly by pathology. The basic information of the included studies is shown in Table II. Other information regarding KLK6 is described in Tables III-V. Furthermore, 2 studies evaluated the SEN of KLK6 with different cut-off values.

SEN, SPE, LR+ and LR- of KLK6. The range of SEN and SPE of KLK6 was 46.6-53.8 and 89.2-92.9\%, respectively. The forest plots of KLK6 with SEN, SPE and the 95\% CI are shown in Figs. 2 and 3. The diagnostic SEN range was 0.47-0.54 (pooled SEN, 0.50) and the SPE range was 0.89-0.93 (pooled SPE, 0.91). The pooled SEN, pooled SPE, pooled LR+ (7.20) and pooled LR- (0.51) of KLK6 are shown in Table VI. The sROC curve of KLK6 is shown in Fig. 4. The AUC and Q* index of KLK6 was 0.86 and 0.79 , respectively.

Possible sources of heterogeneity. The KLK6 of the sROC curve shows that the plane scatter plot does not have a typical 'shoulder arm-shaped' style (Fig. 4), while the Spearman correlation coefficient was 0.180 and $\mathrm{P}=0.670$, which indicates that there is no heterogeneity from threshold effects. However, the pool SEN $\left(I^{2}=83.1 \%\right)$ and SPE $\left(I^{2}=91.7 \%\right)$ indicates high levels of heterogeneity. The control group, the test method, the assay kit, a cut-off value, the mean age and the study size were considered for the source of the heterogeneity (14).

\section{Discussion}

Ovarian cancer is a leading cause of cancer-associated fatality among women in Western Europe and the USA, and it has the highest mortality rate of all gynecological malignancies (18). 
Table I. Quality assessment of the 5 eligible studies using QUADAS2.

\begin{tabular}{|c|c|c|c|c|c|c|c|c|}
\hline \multirow[b]{2}{*}{ Authors, year } & \multicolumn{4}{|c|}{ Risk of bias } & \multicolumn{3}{|c|}{ Applicability concerns } & \multirow[b]{2}{*}{ Refs. } \\
\hline & $\begin{array}{l}\text { Patient } \\
\text { selection }\end{array}$ & $\begin{array}{c}\text { Index } \\
\text { test }\end{array}$ & $\begin{array}{c}\text { Reference } \\
\text { standard }\end{array}$ & $\begin{array}{l}\text { Flow and } \\
\text { timing }\end{array}$ & $\begin{array}{l}\text { Patient } \\
\text { selection }\end{array}$ & $\begin{array}{c}\text { Index } \\
\text { test }\end{array}$ & $\begin{array}{l}\text { Reference } \\
\text { standard }\end{array}$ & \\
\hline Bandiera et al, 2013 & High & Low & Unknown & High & High & Low & Low & $(15)$ \\
\hline Diamandis et al, 2003 & High & Unknown & Low & Unknown & High & Unknown & Low & $(10)$ \\
\hline Diamandis et al, 2000 & High & High & Unknown & Low & High & Unknown & Unknown & (8) \\
\hline Koh et al, 2012 & High & High & Unknown & High & High & Low & Unknown & $(16)$ \\
\hline El Sherbini et al, 2011 & High & Low & Unknown & Low & Unknown & Unknown & Low & $(17)$ \\
\hline
\end{tabular}

Table II. Main characteristics of the 5 studies.

\begin{tabular}{|c|c|c|c|c|c|c|c|}
\hline \multirow[b]{2}{*}{ Authors, year } & \multirow[b]{2}{*}{ Country } & \multirow[b]{2}{*}{ Test method } & \multirow[b]{2}{*}{ Study size, n } & \multirow[b]{2}{*}{$\mathrm{OC}, \mathrm{n}$} & \multicolumn{2}{|c|}{ Control group, $\mathrm{n}$} & \multirow[b]{2}{*}{ Refs } \\
\hline & & & & & $\mathrm{BD}$ & Healthy & \\
\hline Bandiera et al, 2013 & Italy & ELISA & 180 & 60 & 60 & 60 & (15) \\
\hline Diamandis et al, 2003 & Canada & ELISA & 384 & 146 & 141 & 97 & $(10)$ \\
\hline Diamandis et al, 2000 & Canada & ELISA & 161 & 80 & - & 81 & (8) \\
\hline Koh et al, 2012 & Singapore & ELISA & 328 & 172 & 156 & - & $(16)$ \\
\hline El Sherbini et al, 2011 & Egypt & ELISA & 97 & 27 & 63 & 7 & $(17)$ \\
\hline
\end{tabular}

$\mathrm{BD}$, benign disease; OC, ovarian cancer; ELISA, enzyme-linked immunosorbent assay.

Table III. Mean age and disease stage of the samples.

\begin{tabular}{|c|c|c|c|c|c|c|}
\hline \multirow[b]{2}{*}{ Authors, year } & \multicolumn{3}{|c|}{ Age, years } & \multicolumn{3}{|c|}{ Disease stage, $\mathrm{n}$} \\
\hline & Healthy & $\mathrm{BD}$ & $\mathrm{OC}$ & Early stages (I and II) & Late stages (III and IV) & Refs. \\
\hline Bandiera et al, 2013 & 52 & 46 & 56 & 43 & 103 & $(15)$ \\
\hline Diamandis et al, 2003 & - & - & - & - & - & $(10)$ \\
\hline Diamandis et al, 2000 & 50 & 58 & 62 & 17 & 43 & $(8)$ \\
\hline Koh et al, 2012 & - & 38.3 & 45.5 & 72 & 100 & $(16)$ \\
\hline El Sherbini et al, 2011 & 40 & 36 & 43 & 17 & 10 & $(17)$ \\
\hline
\end{tabular}

$\mathrm{BD}$, benign disease; $\mathrm{OC}$, ovarian cancer.

Table IV. Types of ovarian cancer of the cohorts in the study.

\begin{tabular}{|c|c|c|c|c|c|c|}
\hline Authors, year & Mucinous, $\mathrm{n}$ & Serous, $\mathrm{n}$ & Endometrioid, $\mathrm{n}$ & Clear cell, $\mathrm{n}$ & Others, $\mathrm{n}$ & Refs. \\
\hline Bandiera et al, 2013 & 22 & 74 & 15 & & 35 & $(15)$ \\
\hline Diamandis et al, 2003 & - & - & - & - & - & $(10)$ \\
\hline Diamandis et al, 2000 & 3 & 19 & 9 & 8 & 21 & $(8)$ \\
\hline Koh et al, 2012 & 50 & 39 & 17 & 7 & 59 & $(16)$ \\
\hline El Sherbini et al, 2011 & - & - & - & - & - & $(17)$ \\
\hline
\end{tabular}

Diagnosis of ovarian cancer lacks noninvasive tests or clear biomarkers, and the majority of patients are diagnosed at advanced stages, which have a poor prognosis. The discovery of new ovarian cancer biomarkers for early diagnosis, prognosis, monitoring and prediction of therapeutic response may contribute to improved clinical outcomes. Therefore, novel 
Table V. Cut-off value, TP, FP, FN, TN, SEN and SPE of KLK6.

\begin{tabular}{|c|c|c|c|c|c|c|c|c|}
\hline Authors, year & Cut off, $\mu \mathrm{g} / \mathrm{l}$ & $\mathrm{TP}, \mathrm{n}$ & $\mathrm{FP}, \mathrm{n}$ & $\mathrm{FN}, \mathrm{n}$ & $\mathrm{TN}, \mathrm{n}$ & SEN, \% & SPE, $\%$ & Refs. \\
\hline \multirow[t]{3}{*}{ Bandiera et al, 2013} & 10.79 & 42 & 7 & 18 & 53 & 70 & 90 & \multirow[t]{3}{*}{$(15)$} \\
\hline & 15.31 & 36 & 1 & 24 & 59 & 60 & 96 & \\
\hline & 22.94 & 23 & 1 & 37 & 59 & 38 & 100 & \\
\hline \multirow[t]{2}{*}{ Diamandis et al, 2003} & 4.2 & 76 & 24 & 70 & 214 & 52 & 90 & \multirow[t]{2}{*}{$(10)$} \\
\hline & 4.4 & 69 & 12 & 77 & 226 & 47 & 95 & \\
\hline Diamandis et al, 2000 & 15 & 53 & 0 & 27 & 81 & 66 & 100 & (8) \\
\hline Koh et al, 2012 & 6 & 61 & 12 & 111 & 144 & 35.2 & 92.1 & (16) \\
\hline El Sherbini et al, 2011 & 3.145 & 17 & 27 & 10 & 36 & 64 & 56.82 & (17) \\
\hline
\end{tabular}

FN, false-negative; FP, false-positive; KLK6, kallikrein-related peptidase 6; TN, true-negative; TP, true-positive; SEN, sensitivity; SPE, specificity.

Table VI. Pooled sensitivity, pooled specificity, pooled LR+, pooled LR-, AUC and Q* of KLK6.

\begin{tabular}{|c|c|c|c|c|c|c|}
\hline Gene & $\begin{array}{l}\text { Pooled sensitivity, \% } \\
\text { (95\% CI) }\end{array}$ & $\begin{array}{l}\text { Pooled specificity, \% } \\
\text { (95\% CI })\end{array}$ & $\begin{array}{l}\text { Pooled LR+ } \\
\quad(95 \% \mathrm{CI})\end{array}$ & $\begin{array}{l}\text { Pooled LR- } \\
(95 \% \mathrm{CI})\end{array}$ & AUC & $\mathrm{Q}^{*}$ \\
\hline$K L K 6$ & $50(47-54)$ & $91(89-93)$ & $7.20(3.34-15.51)$ & $0.51(0.43-0.62)$ & 0.855 & 0.786 \\
\hline
\end{tabular}

Control group included benign disease patients and healthy controls. AUC, area under the summary receiver operating characteristic curve; CI, confidence interval; KLK6, kallikrein-related peptidase 6; LR+, positive-likelihood ratio; LR-, negative-likelihood ratio.

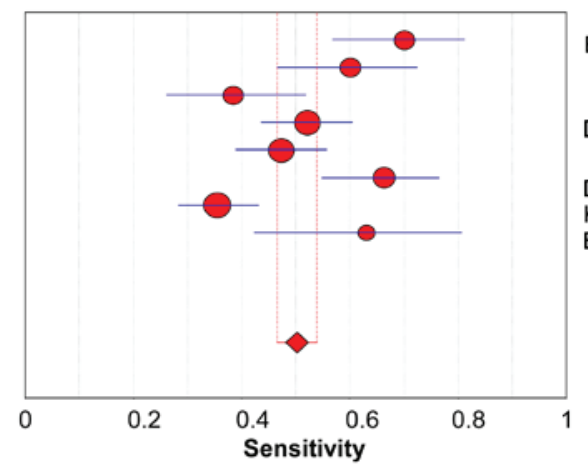

$\begin{array}{lll}\text { Bandiera et al (15) } & 0.70 & (0.57-0.81) \\ & 0.60 & (0.47-0.72) \\ \text { Diamandis et al (10) } & 0.38 & (0.26-0.52) \\ & 0.52 & (0.44-0.60) \\ \text { Diamandis et al (8) } & 0.47 & (0.39-0.56) \\ \text { Koh et al (16) } & 0.66 & (0.55-0.76) \\ \text { El Sherbini et al (17) } & 0.35 & (0.28-0.43) \\ & 0.63 & (0.42-0.81)\end{array}$

Pooled Sensitivity $=0.50(0.47$ to 0.54$)$

Chi-square $=41.45 ; \mathrm{df}=7(\mathrm{P}<0.0001)$

Inconsistency (I-square) $=83.1 \%$

Figure 2. Forest plot of the sensitivity for KLK6. The red circles represent the sensitivity of one study; the black line shows its confidence interval. If a study reported accuracy data for more than one cut-off, its results are included more than once. CI, confidence interval; KLK6, kallikrein-related peptidase 6.

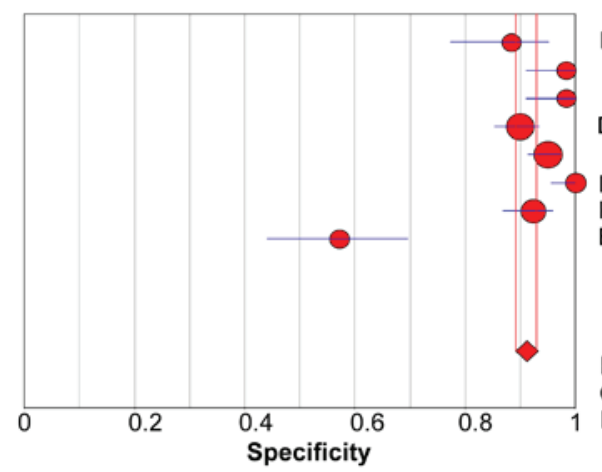

Bandiera et al (15)

Specificity $(95 \% \mathrm{CI})$

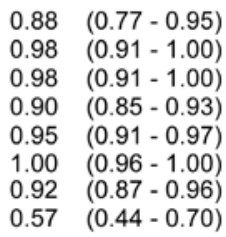

Pooled Specificity $=0.91(0.89$ to 0.93$)$

Chi-square $=84.03 ; \mathrm{df}=7 \quad(\mathrm{P}<0.0001)$

Inconsistency (I-square) $=91.7 \%$

Figure 3. Forest plot of the specificity for KLK6. The red circles represent the specificity of one study; the black line shows its confidence interval. If a study reported accuracy data for more than one cut-off, its result are included more than once. CI, confidence interval; KLK6, kallikrein-related peptidase 6. 


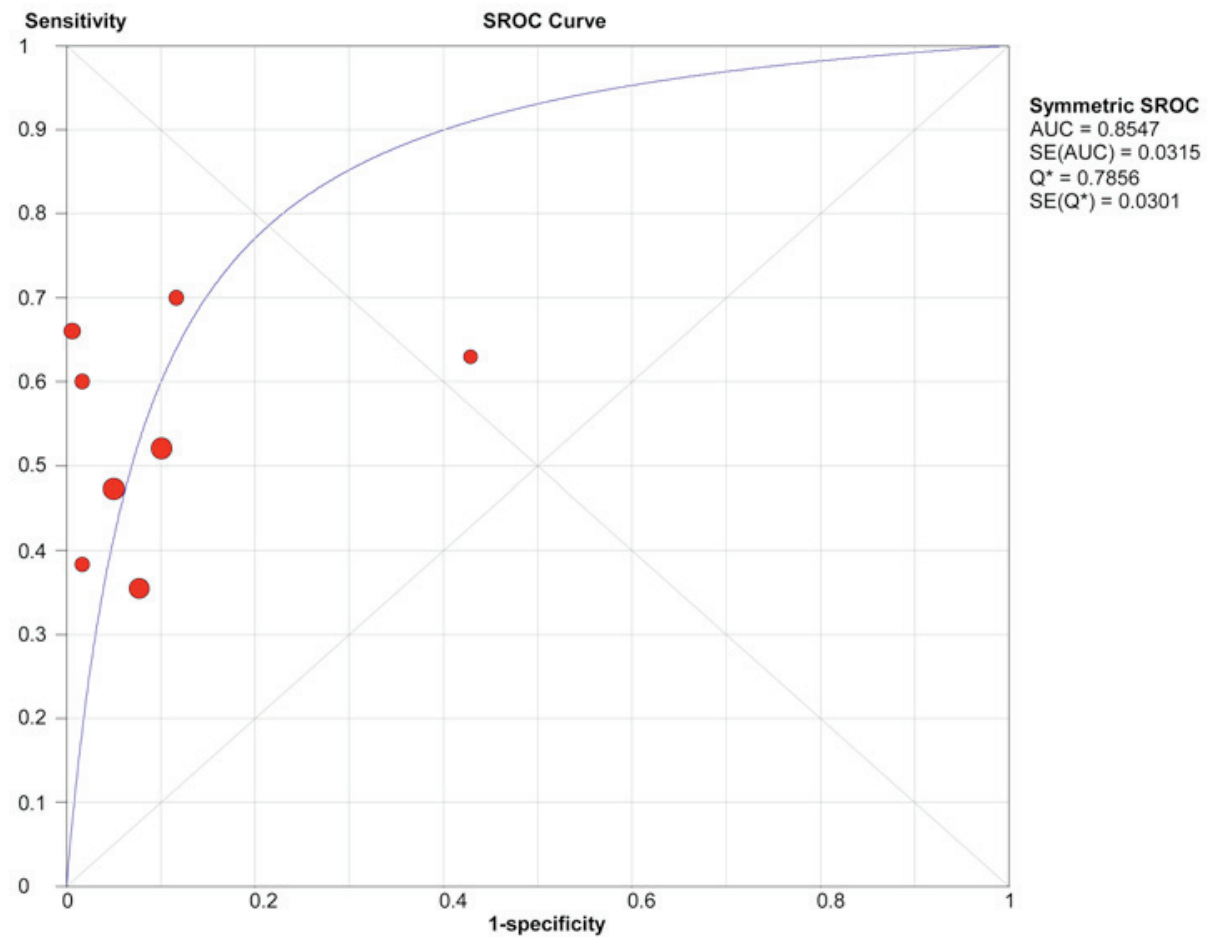

Figure 4. sROC and AUC of kallikrein-related peptidase 6. sROC, summary receiver operating characteristic; AUC, area under the curve; SE, standard error.

biomarkers for screening ovarian cancer at early stages are required.

The results of the present meta-analysis showed that the serum KLK6 level could aid in the prediction of the presence of ovarian cancer based on the AUC (AUC=0.85). KLK6 showed SEN 0.50 (0.467 to 0.54) and high SPE 0.91 (0.90 to 0.93). These data indicate that KLK6 is a useful diagnostic marker for ovarian cancer. The associated poor SEN of KLK6 clearly limits its value in its diagnosis of ovarian cancer. However, the high SPE also makes it a potential biomarker of ovarian cancer.

As a traditional ovarian cancer biomarker, CA125 has been used for 20 years. In addition to its low SEN for early disease, CA125 also has low SPE; for example, elevated levels are observed in numerous benign gynecological diseases (18). However, the diagnostic accuracy of CA125 compared to KLK6 in the diagnosis of ovarian cancer was not compared in the present study as CA125 is frequently used clinically and the test results are not always blinded to gynecologists. Among these eligible studies, there were 3 original studies that compared the diagnostic efficiency of KLK6 and CA125. Koh et al (16) found that the SEN and the SPE of KLK6 were lower than those of CA125, but combination of KLK6 and CA125 showed higher diagnostic efficiency. El Sherbini et al (17) and Diamandis et al (9) found that serum KLK6 and may have much lower overall SEN than serum CA125. However, whereas serum KLK6 may improve the SEN of CA125. Mills et al (19) suggested that KLK6 is more specific for ovarian cancer than CA125 as elevations were not observed in BD. Therefore, whether the diagnostic efficiency of KLK6 is superior to that of CA125 remains to be elucidated. However, all these studies evidently supported that KLK6 can improve the diagnostic efficiency of CA125.
The present meta-analysis showed significant heterogeneity between the selected studies. Therefore, due to the heterogeneity, the meta-analysis results should be interpreted with caution. The Spearman correlation coefficient was 0.180 and $\mathrm{P}=0.670$, which indicates that there is no heterogeneity from threshold effects. The following reasons may hamper the statistical analysis of sources of heterogeneity: i) There was no unified cut-off value. ii) The clinical features of the patients, such as the mean age and menopausal status, differed among the included studies. The available data showed that the mean ages ranged from 43 to 62 years. Ages and menopausal status are associated with the occurrence of ovarian cancer. Therefore, it may be a significant source of heterogeneity. iii) The nationality of the study populations differed among the included studies, and the origin of the study populations has a substantial influence on the diagnostic SEN (20). iv) Owing to the small number of patients included in these studies, subgroup analysis was not possible.

The present systematic review has several strengths. In recent decades, a series of studies focused on whether KLK6 was a promising biomarker of ovarian cancer. To the best of our knowledge, this is the first review of the systematic research and meta-analysis to evaluate the diagnostic value of KLK6 for ovarian cancer. All the studies that were published up to April 29, 2015 were searched. There were 485 patients with ovarian cancer, 420 benign cysts and 245 healthy controls involved in the present study. Study selection, data extraction, evaluation of the risk of bias and strength of inferences for each outcome were performed by two investigators independently, to reduce the risk of selection bias. However, there were several limitations in the meta-analysis. First, the number of the included studies was too small. Second, the difference in the mean age and menopausal status may bias the results. 
Third, the limitation of the present meta-analysis was that there was no unified cut-off value.

In conclusion, the high SPE of human KLK6 shows that it is a potential novel biomarker for ovarian cancer. It can evidently improve diagnostic efficiency of ovarian cancer and KLK6 can improve the diagnostic accuracy of CA125. However, according to the present meta-analysis, KLK6 does not meet the standard of an independent diagnostic biomarker. A combined panel of CA125 and KLK6 showed a high diagnostic efficiency for advanced ovarian cancer. Due to the limitations in the present meta-analysis, additional studies are required to assess the diagnostic accuracy of KLK6 in the future.

\section{References}

1. Siegel R, Ward E, Brawley O and Jemal A: Cancer statistics, 2011: The impact of eliminating socioeconomic and racial disparities on premature cancer deaths. CA Cancer J Clin 61: 212-236, 2011.

2. Rosenthal AN and Jacobs IJ: The role of CA 125 in screening for ovarian cancer. Int J Biol Markers 13: 216-220, 1998.

3. Maggino $T$ and Gadducci A: Serum markers as prognostic factors in epithelial ovarian cancer: An overview. Eur J Gynaecol Oncol 21: 64-69, 2000.

4. Bast RC Jr, Xu FJ, Yu YH, Barnhill S, Zhang Z and Mills GB: CA 125: The past and the future. Int J Biol Markers 13: 179-187, 1998.

5. Bast RC Jr, Klug TL, St John E, Jenison E, Niloff JM, Lazarus H, Berkowitz RS, Leavitt T, Griffiths CT, Parker L, et al: A radioimmunoassay using a monoclonal antibody to monitor the course of epithelial ovarian cancer. N Engl J Med 309: 883-887, 1983.

6. Lundwall A, Band V, Blaber M, Clements JA, Courty Y, Diamandis EP, Fritz H, Lilja H, Malm J, Maltais LJ, et al: A comprehensive nomenclature for serine proteases with homology to tissue kallikreins. Biol Chem 387: 637-641, 2006.

7. Yousef GM and Diamandis EP: The new human tissue kallikrein gene family: Structure, function, and association to disease Endocr Rev 22: 184-204, 2001.

8. Diamandis EP, Yousef GM, Soosaipillai AR and Bunting P: Human kallikrein 6 (zyme/protease M/neurosin): A new serum biomarker of ovarian carcinoma. Clin Biochem 33: 579-583, 2000 .
9. Diamandis EP, Yousef GM, Soosaipillai AR, Grass L, Porter A, Little S and Sotiropoulou G: Immunofluorometric assay of human kallikrein 6 (zyme/protease M/neurosin) and preliminary clinical applications. Clin Biochem 33: 369-375, 2000.

10. Diamandis EP, Scorilas A, Fracchioli S, Van Gramberen M, De Bruijn H, Henrik A, Soosaipillai A, Grass L, Yousef GM, Stenman UH, et al: Human kallikrein 6 (hK6): A new potential serum biomarker for diagnosis and prognosis of ovarian carcinoma. J Clin Oncol 21: 1035-1043, 2003.

11. Luo LY, Bunting P, Scorilas A and Diamandis EP. Human kallikrein 10: A novel tumor marker for ovarian carcinoma? Clinica Chim Acta 306: 111-118, 2001.

12. Chen X, Li WL, Zhang YL, Wu Q, Guo YM and Bai ZL: Meta-analysis of quantitative diffusion-weighted MR imaging in the differential diagnosis of breast lesions. BMC Cancer 10: 693, 2010.

13. Whiting PF, Rutjes AW, Westwood ME, Mallett S, Deeks JJ, Reitsma JB, Leeflang MM, Sterne JA and Bossuyt PM; QUADAS-2 Group: QUADAS-2: A revised tool for the quality assessment of diagnostic accuracy studies. Ann Intern Med 155: 529-536, 2011.

14. Zamora J, Abraira V, Muriel A, Khan K and Coomarasamy A: Meta-DiSc: A software for meta-analysis of test accuracy data. BMC Med Res Methodol 6: 31, 2006.

15. Bandiera E, Zanotti L, Fabricio AS, Bucca E, Squarcina E, Romani C, Tassi R, Bignotti E, Todeschini P, Tognon G, et al: Cancer antigen 125, human epididymis 4, kallikrein 6, osteopontin and soluble mesothelin-related peptide immunocomplexed with immunoglobulin $\mathrm{M}$ in epithelial ovarian cancer diagnosis. Clin Chem Lab Med 51: 1815-1824, 2013.

16. Koh SC, Huak CY, Lutan D, Marpuang J, Ketut S, Budiana NG, Saleh AZ, Aziz MF, Winarto H, Pradjatmo H, et al: Combined panel of serum human tissue kallikreins and CA-125 for the detection of epithelial ovarian cancer. J Gynecol Oncol 23: 175-181, 2012.

17. El Sherbini MA, Sallam MM, Shaban EA and El-Shalakany AH: Diagnostic value of serum kallikrein-related peptidases 6 and 10 versus CA125 in ovarian cancer. IInt J Gynecol Cancer 21: 625-632, 2011.

18. Campos SM and Ghosh S: A current review of targeted therapeutics for ovarian cancer. J Oncol 2010: 149362, 2010.

19. Mills GB, Bast RC Jr and Srivastava S: Future for ovarian cancer screening: Novel markers from emerging technologies of transcriptional profiling and proteomics. J Natl Cancer Inst 93: 1437-1439, 2001.

20. Whiting P, Rutjes AW, Reitsma JB, Glas AS, Bossuyt PM and Kleijnen J: Sources of variation and bias in studies of diagnostic accuracy: A systematic review. Ann Intern Med 140: 189-202, 2004. 